\title{
Characteristics and Evaluation of Thermal Comfort Around River in Banjarmasin City
}

\author{
Akbar Rahman \\ Department of Civil Engineering and Architecture \\ Saga University Japan \\ Department of Engineering \\ Lambung Mangkurat University Indonesia
}

\author{
Shoichi Kojima \\ Department of Civil Engineering and Architecture \\ Saga University Japan
}

\begin{abstract}
The density of buildings in a city has affected the urban microclimate. The urban area gets hotter than the rural area. Characteristics of nature and climate need special attention. The design of the house determines the effect on indoor and outdoor thermal comfort. This research was conducted in two sub-districts or three urban villages in Banjarmasin. The subdistricts are Central Banjarmasin sub-district and the East Banjarmasin sub district. Results, Thermal comfort of Indonesian National Standard (SNI) 03-6572 in open space has a minimum and maximum effective temperature difference, between open space without vegetation and vegetation. Vegetation planted around the building has an effect on the buildings thermal environment. Vegetation around rivers and buildings can lower the average effective temperature by $0.7^{\circ} \mathrm{C}$ ET. At a minimum, the effective temperature difference between green open space and open space without vegetation is $1^{\circ} \mathrm{C}$ ET. The thermal comfort condition in open space without vegetation in uncomfortable condition while vegetated still in a comfortable zone. The indoor effective temperature has differences between riverside buildings and settlements are $1^{\circ} \mathrm{C}$ ET. Conversely, at maximum effective temperature, open space without vegetation is higher than $2^{\circ} \mathrm{C}$ ET compared to open space with vegetation. The thermal comfort condition in buildings with ventilation and ceiling are better, but the buildings with zinc material for the roof are hotter.
\end{abstract}

Keywords - characteristic; evaluation; thermal environment; thermal comfort.

\section{INTRODUCTION}

Climate and environmental aspects are one of the things that affect architectural products [1]. The climate, or average weather, is primarily a function of the sun. The word "climate" comes from the Greek "Klima", which means the slope of the earth with respect to the sun. The Greeks realized that climate is largely a function of sun angles (latitude) and, therefore, they divided the world into the tropic, temperate, and arctic zones [2]. The climate of a place is known to be at least 10-30 years of average annual weather such as air humidity, temperature, wind patterns and rainfall. Another indication that can be used as a determinant of climate type is vegetation that dominates a region, such as tropical rainforest, deciduous forest, and coniferous forest. Tropical is a word derived from the Greek word "tropikos" which means a return line are latitude $23^{\circ} 27^{\prime}$ North and South. The tropical region is defined as an area located between the $20^{\circ}$ isotherm line next to the North and South earths [3].

The city as a gathering place that interacts for a particular purpose has influenced the shape and visual of the city. The increasing population living in a city, causing the complexity of the problem, is no exception to the architecture and urban design. The density of buildings in a city has affected the urban microclimate. Urban get hotter than rural areas [4]. Urban planners need to pay attention to several aspects related to the solution to the design of the humid tropics. The relationship between design and rules is the first half of what architects and planners need to know, that is, how design meets the requirements of the rules [5]. For example, urban design should pay attention to the comfort of pedestrians. Pedestrians do not need to use an umbrella, shelter when it rains and the sun shines. Pedestrian way should be provided with corridors that are protected at the top. Buildings must be connected, so the activity does not stop when it rains or the sun shines (Figure 1.a) [6].
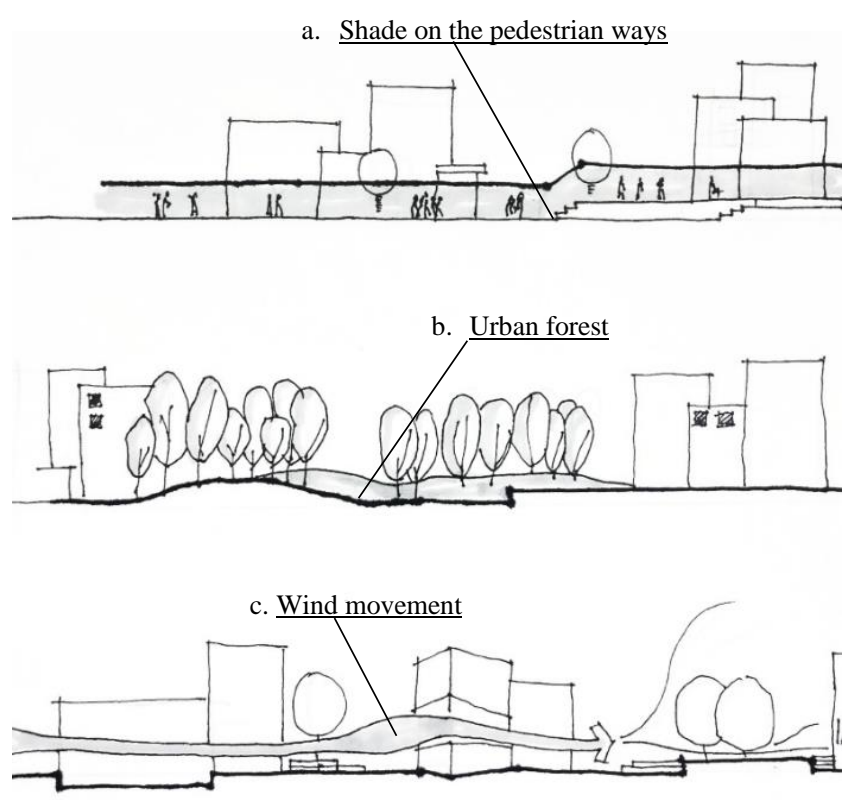

Fig 1. Factors to beconsidered in urban design

Creating urban forest will minimize heat absorption on the outdoor surface. The goal is the urban forest to anticipate high temperatures. Direct sun exposure to solid surfaces (asphalt, concrete and other) should be avoided. The solid surface needs to be protected by trees, grasses, plants or vegetation. Vegetation can absorb and reflect the heat. So the area covered by vegetation has a relatively low air temperature compared to the open area (Figure 1.b). The hard materials used as surface coatings (asphalt, concrete and others) will absorb much heat, but then the heat will reflect into the air, resulting in the warming of the surrounding air $[7,8]$. Thus, the concept of 
greening the city evenly and thoroughly, so that the air temperature is not too high that can affect comfort.

The building mass arrangement by optimizing the airflow around the building, needs to be a concern. One way to improve thermal comfort in humid tropical climates is to optimize the airflow around (outdoor) and indoor buildings. Wind movement will be better in open space if airflow is not blocked [6]. In the concept of arrangement of building mass, open space between buildings is important so that the air flow around the building. It is expected to be utilized by buildings to create cross ventilation so as to lower indoor air temperatures (Figure 1.c)

\section{A. Thermal Comfort in Public Space: Pedestrian Ways and Public House}

The elements that affect comfort in a pedestrian are circulation, accessibility, characteristics of nature and climate, safety, cleanliness, and beauty [9]. Characteristics of nature and climate need special attention. Indonesia's condition, which consists of islands that are surrounded by oceans, causes a humid environment. Hot-humid conditions affect the comfort of pedestrians. The elements of humid tropical climate that affect the comfort level are solar radiation, temperature, humidity, rainfall, and wind movement. In addition, vegetation also affects the comfort of pedestrians. Vegetation serves as aesthetic, also serves as a shade, wind barrier into the building and barrier of solar thermal radiation [10]. For thermal comfort of pedestrians, it takes pedestrians support facilities (street furniture), as follows:

- Ground Cover on pedestrian ways can be hard material and soft material. Hard materials, for example are paving, concrete, brick, stone, and asphalt, while soft materials such as clay and grass. Planning ground cover depends on the function and type of pedestrian ways.

- Shelter, shaped linear as a corridor or sitting group whose function can be a place to rest, take shelter from the heat or rain, as well as for public transportation shuttles.

- Vegetation in the pedestrian ways can serve as a barrier by road vehicle or parking. Barrier also can reduce noise, vehicle pollution and shade in the rain. In addition, vegetation also serves to reduce solar heat radiation.

\section{B. Correlation of Thermal Comfort with the House Design}

The design of the house determines the effect on indoor and outdoor thermal comfort. Thermal comfort of the building can be achieved if the following things are considered:

- Locations are important in building design, especially in humid tropical climates. In the area around the river or sea have high humidity especially in the morning. Temperatures in the mainland heat faster than the watery areas [11].
- Form and building components are important things to consider in order to achieve thermal comfort in buildings. A building that maximizes sunlight for natural lighting, but can still control its heat for thermal comfort. The form can also affect the flow of wind for effective cross-ventilation or high ceilings in the room [12]. Building components also affect the thermal comfort of buildings, such as windows, roofs, and walls. Moreover, one thing that most affects the thermal environment is the material [13]

\section{RESEARCH METHODS}

A. Description of Location and Places of Research

This research was conducted in two sub districts or three urban villages in Banjarmasin. The sub-districts are Central Banjarmasin sub-district and the East Banjarmasin subdistrict. The village located in the Central Banjarmasin subdistrict, namely: Gadang Village and Sasirangan Village, while the research location in East Banjarmasin is Bilu Village. The villages are located around the Martapura River. The activities of residents in Bilu Village are heavily influenced by the river. The research was conducted in three places: 1) Siring Tendean-Gadang Village, 2) Sasirangan Village and 3) Bilu Village. (Figure 2).

Siring Tendean is a public space that becomes a new tourist destination in Banjarmasin. This area was once a slum settlement area around the Martapura River. Since 2008, the government of Banjarmasin City has made a revamping of the city area through the arrangement of slum areas on the banks of the river, namely the construction of Siring, construction of Menara and renovation of Anno's house [14,15,16]. Siring Tendean has become a new landmark in Banjarmasin City with Tower and open space. To attract tourists visiting this area, the city government of Banjarmasin prepares support activities such as the restaurant at Anno's house and 'create' new floating market.

Sasirangan village is a residential area of Seberang Mesjid located around the Martapura River. This area has become a tourist destination in the Banjarmasin city, because this area produces sasirangan fabrics. In addition to being the center of the traditional clothing industry, this area also has a traditional house type that is a floating house. This floating house has a unique architectural building construction, because it can float in the river. The uniqueness of the floating house attract visitors to the Sasirangan Village and also becomes the center of the study of the Banjar traditional house, for academics and students. Since 2015, the City Government has made improvements in the area, especially the slums on the errand on the Martapura River. The goal is that visitors who come to the Sasirangan village will feel comfortable. The City Government has renovated floating houses for better and more accessible to visitors. In addition, the houses are arranged and tidied up construction and re-painted with sasirangan motifs as characteristic of Sasirangan village.

Bilu village is a traditional settlement in Banjarmasin City. This is evident from the orientation of the house facing the river. Along the banks of the river there is a pedestrian street, local people call it 'Titian'. This pedestrian way has been 
around since the beginning of the settlement was built. Pedestrian way in Bilu village is also used by residents as a place of social interaction, so this pedestrian way also becomes a public space area. Since 2016, this settlement has been arranged by the City Government. The pedestrian way is being renovated and the river-oriented houses are painted in green to make it look attractive, clean and no more slums. Today, this village is known as the "green" village.

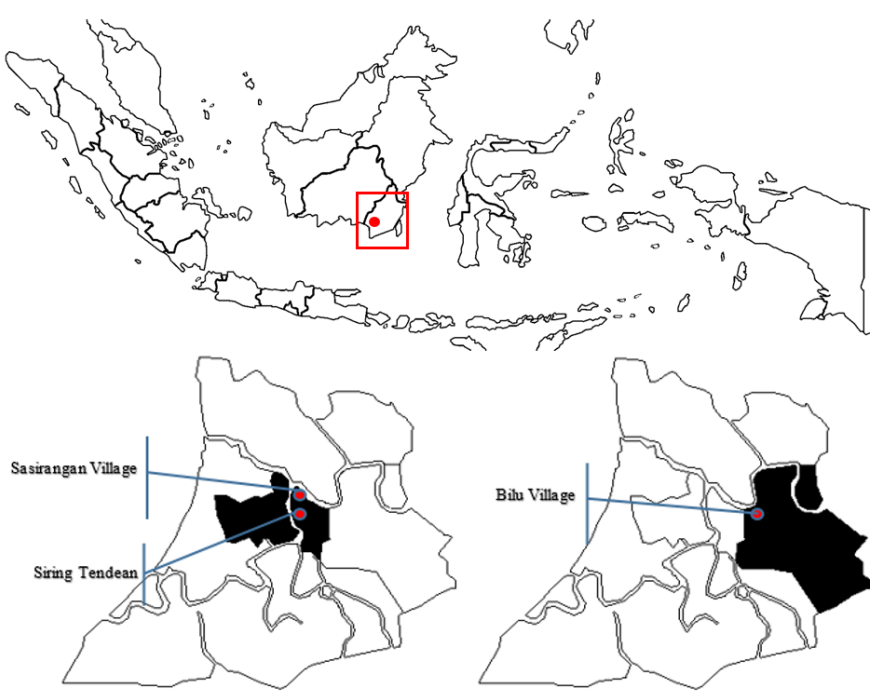

Fig 2. Research location(in Indonesia-Banjarmasin and places,(by)) subdistrict of Center Banjarmasin, b) subdistrict of East banjarmasin

\section{B. Research Variables and Measurement Procedure}

Thermal comfort is that condition of mind which expresses satisfaction with the thermal environment and is assessed by subjective evaluation. Because there are large variations, both physiologically and psychologically, from person to person, it is difficult to satisfy everyone in a space. The environmental conditions required for comfort are not same for everyone. Thermal sensation: a conscious feeling commonly graded into the categories, cold, cool, slightly cool, neutral, slightly warm, warm, and hot; it requires subjective evaluation [17].

The Government of Indonesia, through the Department of Kementrian PUPR has issued thermal comfort standards of space in buildings. The National Standardization Agency of Indonesia (BSN) issued a national standard ventilation system and energy conservation $[18,19]$. This standard is intended as a guide for planning, building and building management.

Indonesia already has a national standard for thermal comfort that is SNI-03-6572 and SNI 6390. The SNI 03-6572 with the effective temperature (ET) of $20.5^{\circ} \mathrm{C}$ to $27.1^{\circ} \mathrm{C}$ and SNI 6390 with the operative temperature $\left(\mathrm{T}_{\text {op }}\right) 24^{\mathrm{O}} \mathrm{C}$ to $27^{\circ} \mathrm{C}$. In this study, thermal comfort SNI 03-6572 is used, because it uses an effective temperature that has many variables: temperature, relative humidity, and wind velocity. Determination of the comfort zone is not only seen from a dry bulb temperature, but also pay attention to humidity and wind velocity $[14,20]$. The thermal comfort index at an effective temperature for humid tropics can be divided into:

- Cool comfort, between the effective temperature of $20.5^{\circ} \mathrm{C}$ to $22.8^{\mathrm{O}} \mathrm{C}$
- Optimal comfort, between the effective temperature of $22.8^{\mathrm{O}} \mathrm{C}$ to $25.8^{\mathrm{O}} \mathrm{C}$.

- Warm comfort, between the effective temperature of $25.8^{\circ} \mathrm{C}$ to $27.1^{\circ} \mathrm{C}$.

Field measurements were conducted in three locations from $11^{\text {th }}$ to $27^{\text {th }}$ September 2016 with the following details: Bilu Village from $11^{\text {th }}$ to $15^{\text {th }}$ September 2016 , Sasirangan Village of $18^{\text {th }}$ to $21^{\text {th }}$ September 2016 and Siring Tendean from $25^{\text {th }}$ to $27^{\text {th }}$ September 2016. Update data and evaluation of building construction on $12^{\text {th }}$ to $20^{\text {th }}$ March 2018. Measurement period and instruments can be seen in Table 1.

TABLE 1. Measurement Period and Instrument

\begin{tabular}{|c|c|}
\hline Description & Explanation \\
\hline Measurement items & $\begin{array}{l}\text { Dry bulb temperature, relative humidity, and } \\
\text { wind velocity }\end{array}$ \\
\hline Measuring instruments & $\begin{array}{l}\text { Data logger-4HC for temperature and } \\
\text { humidity } \\
\text { Extech AN100 for air velocity }\end{array}$ \\
\hline $\begin{array}{l}\text { Calculate of wet bulb } \\
\text { temperature }\end{array}$ & Psychometric charts \\
\hline $\begin{array}{l}\text { Calculate of effective } \\
\text { temperature }\end{array}$ & Diagrams effective temperature \\
\hline Measurement period & $\begin{array}{l}11^{\text {th }}-27^{\text {th }} \text { September } 2016 \\
\text { Time: 6:00 am to 05:00 am (every hour/24 } \\
\text { hours) }\end{array}$ \\
\hline $\begin{array}{l}\text { Update data and building } \\
\text { evaluation }\end{array}$ & $12^{\text {th }}-20^{\text {th }}$ March 2018 \\
\hline $\begin{array}{l}\text { High measuring instruments } \\
\text { from the ground/floor }\end{array}$ & $150 \mathrm{~cm}$ \\
\hline Measuring points & $\begin{array}{l}\text { Indoor and outdoor: } \\
\text { 1. Siring Tendean (1a,1b,1c,1d,1e) } \\
\text { 2. Sasirangan village }(2 \mathrm{a}, 2 \mathrm{~b}, 2 \mathrm{c}, 2 \mathrm{~d}, 2 \mathrm{e}, 2 \mathrm{f}) \\
\text { 3. Billu village (3a, 3b, 3c, 3d, 3e, 3f) }\end{array}$ \\
\hline Number of measuring points & 2040 points \\
\hline
\end{tabular}

\section{CHARACTERISTICS THERMAL ENVIRONMENT AND THERMAL COMFORT}

\section{A. Siring Tendean}

Siring Tendean is a public space that becomes a new tourist destination in Banjarmasin. This area was once a slum settlement area around the Martapura River. Since 2008, the government of Banjarmasin City has made a revamping of the city area through the arrangement of slum areas on the banks of the river, namely the construction of Siring, construction of Menara and renovation of Anno's house. Currently, Siring Tendean has become a new landmark in the Banjarmasin City. To attract tourists visiting this area, the city government of Banjarmasin prepares support activities such as the restaurant at Anno's house and 'create' new floating market. The floating market is a 'new' traditional market designed for Siring Tendean open space activities (Figure 3 ). So this change can give a positive value [21,22].

The the measurement results in Siring Tendean are difference of maximum dry bulb temperature in indoor between Menara and Anno. The indoor dry bulb temperature in Menara is lower than the outdoor, while the indoor dry bulb temperature in Anno is higher than outdoors. This situation means, the indoor dry bulb temperature in Menara is better and indoor of Anno's house is hotter with dry bulb temperature of $34^{\circ} \mathrm{C}$ (Table 2). The time lag of dry bulb temperature 
between the Menara's house and Anno's house is 1 hour at the same time. The minimum value of Anno's indoor dry bulb temperature is also higher than the Menara and its minimum point is earlier than outdoor. So the house Anno does not have a time lag, while the Menara has a time lag of 2 hours, but if the averaged value of a time lag minimum dry bulb temperature is 30 minutes. The indoor maximum relative humidity in Anno is higher than outdoor, while the indoor maximum relative humidity in Menara is not higher than outdoor. The indoor minimum relative humidity in Menara and Anno are lower than outdoor. Maximum and minimum relative humidity have time lag. The mean time lag of maximum relative humidity is 3.5 hours while the minimum time lag only 30 minutes. The outdoor minimum wet bulb temperature in Menara and Anno are same, but the outdoor maximum wet bulb temperature in Anno is higher than Menara. The wet bulb temperature of time lag only occurs in Menara at maximum value.

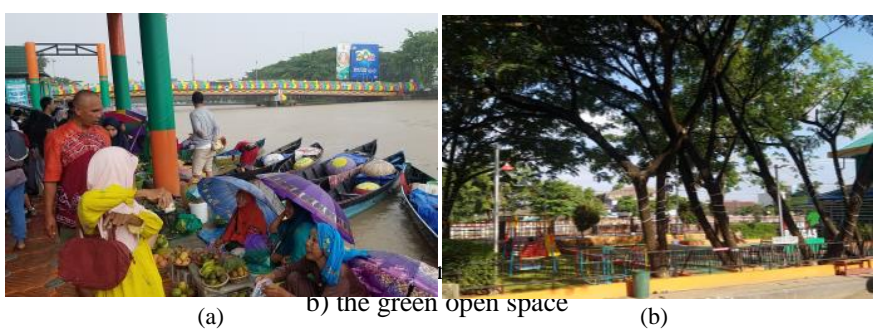

TABLE 2. Difference of Thermal Environment and Time Lag Measurement in Building and Open Space Siring Tendean

\begin{tabular}{|c|c|c|c|c|c|}
\hline \multirow{2}{*}{\multicolumn{2}{|c|}{ Measurment Points }} & \multicolumn{2}{|c|}{ Dry Bulb Temperature } & \multicolumn{2}{|c|}{ Relative Humidity } \\
\hline & & \multirow{2}{*}{$\begin{array}{c}\text { Max/time } \\
30.8^{\circ} \mathrm{C} / 2 \mathrm{PM}\end{array}$} & \multirow{2}{*}{$\begin{array}{c}\text { Min/time } \\
27.2^{\circ} \mathrm{C} / 7 \mathrm{AM}\end{array}$} & \multirow{2}{*}{$\begin{array}{l}\text { Max/time } \\
\text { 75\%/9AM }\end{array}$} & \multirow{2}{*}{$\begin{array}{c}\text { Min/time } \\
60.1 \% / 4 \mathrm{PM}\end{array}$} \\
\hline & 1.a. (1) & & & & \\
\hline$\Xi g$ & 1.b. (1) & $34^{\circ} \mathrm{C} / 2 \mathrm{PM}$ & $28^{\circ} \mathrm{C} / 5 \mathrm{AM}$ & $80.7 \% / 8 \mathrm{AM}$ & $58.8 \% / 4 \mathrm{PM}$ \\
\hline \multirow{5}{*}{ 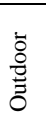 } & 1.a. (2) & $33.8^{\circ} \mathrm{C} / 1 \mathrm{PM}$ & $25.5^{\circ} \mathrm{C} / 5 \mathrm{AM}$ & $79.1 \% / 5 \mathrm{AM}$ & $61.3 \% / 3 \mathrm{PM}$ \\
\hline & 1.b. (2) & $33.2^{\circ} \mathrm{C} / 1 \mathrm{PM}$ & $25.5^{\circ} \mathrm{C} / 6 \mathrm{AM}$ & $79.5 \% / 5 \mathrm{AM}$ & $61.5 \% / 2 \mathrm{PM}$ \\
\hline & 1.c. (3) & $34^{\circ} \mathrm{C} / 3 \mathrm{PM}$ & $24.7^{\circ} \mathrm{C} / 5 \mathrm{AM}$ & $85.4 \% / 5 \mathrm{AM}$ & $62.9 \% / 3 \mathrm{PM}$ \\
\hline & 1.d. (4) & $32.8^{\circ} \mathrm{C} / 4 \mathrm{PM}$ & $25.6^{\circ} \mathrm{C} / 5 \mathrm{AM}$ & $85.1 \% / 8 \mathrm{AM}$ & $66 \% / 4 \mathrm{PM}$ \\
\hline & 1.e. (4) & $31.9^{\circ} \mathrm{C} / 4 \mathrm{PM}$ & $25.2^{\circ} \mathrm{C} / 6 \mathrm{AM}$ & $85.5 \% / 8 \mathrm{AM}$ & $65.5 \% / 5 \mathrm{PM}$ \\
\hline \multirow{10}{*}{$\stackrel{\text { ָี }}{\sum}$} & Indoor (1) & $32.4^{\circ} \mathrm{C} / 2 \mathrm{PM}$ & $27.6^{\circ} \mathrm{C} / 6 \mathrm{AM}$ & $77.9 \% / 8.30 \mathrm{AM}$ & $59.5 \% / 4 \mathrm{PM}$ \\
\hline & Outdoor (2) & $33.5^{\circ} \mathrm{C} / 1 \mathrm{PM}$ & $25.5^{\circ} \mathrm{C} / 5: 30 \mathrm{AM}$ & $79.3 \% / 5 \mathrm{AM}$ & $61.4 \% / 3.30 \mathrm{PM}$ \\
\hline & Outdoor (3) & $34^{\circ} \mathrm{C} / 3 \mathrm{PM}$ & $24.7^{\circ} \mathrm{C} / 5 \mathrm{AM}$ & $85.4 \% / 5 \mathrm{AM}$ & $62.9 \% / 3 \mathrm{PM}$ \\
\hline & Outdoor (4) & $32.4^{\circ} \mathrm{C} / 4 \mathrm{PM}$ & $25.4^{\circ} \mathrm{C} / 5: 30 \mathrm{AM}$ & $85.3 \% / 8 \mathrm{AM}$ & $65.8 \% / 4: 30 \mathrm{PM}$ \\
\hline & Difference (1)(2) & $1.1^{\circ} \mathrm{C}$ & $2.1^{\circ} \mathrm{C}$ & $1.40 \%$ & $2.70 \%$ \\
\hline & Difference (1)(3) & $1.6^{\circ} \mathrm{C}$ & $2.9^{\circ} \mathrm{C}$ & $7.50 \%$ & $3.40 \%$ \\
\hline & Difference (1)(4) & 0 & $2.2^{\circ} \mathrm{C}$ & $7.40 \%$ & $6.30 \%$ \\
\hline & Time lag (1)(2) & $1 \mathrm{~h}$ & $0.5 \mathrm{~h}$ & $3.5 \mathrm{~h}$ & $0.5 \mathrm{~h}$ \\
\hline & Time lag (1)(3) & 0 & $1 \mathrm{~h}$ & $3.5 \mathrm{~h}$ & $1 \mathrm{~h}$ \\
\hline & Time lag (1)(4) & 0 & $0.5 \mathrm{~h}$ & $0.5 \mathrm{~h}$ & 0 \\
\hline
\end{tabular}

The maximum effective temperature in Anno is quite high compared to the Menara, so the Menara is more comfortable than Anno. The difference of maximum effective temperature between outdoor and indoor in Anno's house is about $5.2^{\circ} \mathrm{C}$ ET, when compared to the Menara it is $0.8^{\circ} \mathrm{C}$ ET higher. The time lag occurs at a maximum effective temperature condition of about 3.5 hours while at minimum conditions only 30 minutes. This situation means, the indoor condition is slower to decrease its effective temperature, and this also causes indoor heat. The indoor thermal comfort conditions on the SNI 03-6572 only at the optimal comfort zone, warm comfort zone and uncomfortable in over $27.1^{\circ} \mathrm{C} \mathrm{ET}$. The thermal comfort in open space is good condition. The green open space is better than the open space around the floating market. The maximum dry bulb temperature in the open space around the floating market is high in the afternoon, the difference of the indoor maximum dry bulb temperature is about $1.6^{\circ} \mathrm{C}$. The maximum dry bulb temperature in the green open space and that of indoor buildings are same. The minimum dry bulb temperature in open space around the floating market is also lower than elsewhere in Siring Tendean. The thermal environment and thermal comfort conditions in Siring Tendean can be seen in Table 3 .

TABLE 3. Difference of Thermal Comfort and Time Lag Measurement in Building and Open Space Siring Tendean

\begin{tabular}{|c|c|c|c|c|c|}
\hline \multirow{2}{*}{\multicolumn{2}{|c|}{ Measurment Points }} & \multicolumn{2}{|c|}{ Wet Bulb Temperature } & \multicolumn{2}{|c|}{ Effective Temperature } \\
\hline & & \multirow{2}{*}{$\begin{array}{l}\text { Max/time } \\
25.6^{\circ} \mathrm{C} / 1 \mathrm{PM}\end{array}$} & \multirow{2}{*}{$\begin{array}{c}\text { Min/time } \\
23^{\circ} \mathrm{C} / 6 \mathrm{AM}\end{array}$} & \multirow{2}{*}{$\begin{array}{c}\text { Max/time } \\
27.1^{\circ} \mathrm{C} / 5 \mathrm{PM}\end{array}$} & \multirow{2}{*}{$\begin{array}{c}\text { Min/time } \\
24.2^{\circ} \mathrm{C} / 6 \mathrm{AM}\end{array}$} \\
\hline & 1.a. (1) & & & & \\
\hline$\Xi \underset{\tau}{0}$ & 1.b. (1) & $27.2^{\circ} \mathrm{C} / 11 \mathrm{AM}$ & $23.9^{\circ} \mathrm{C} / 5 \mathrm{AM}$ & $28.8^{\circ} \mathrm{C} / 3 \mathrm{PM}$ & $25^{\circ} \mathrm{C} / 5 \mathrm{AM}$ \\
\hline \multirow{5}{*}{$\frac{\overline{0}}{0}$} & 1.a. (2) & $28.2^{\circ} \mathrm{C} / 12 \mathrm{PM}$ & $22.2^{\circ} \mathrm{C} / 6 \mathrm{AM}$ & $27.2^{\circ} \mathrm{C} / 1 \mathrm{PM}$ & $19.8^{\circ} \mathrm{C} / 6 \mathrm{AM}$ \\
\hline & 1.b. (2) & $27.6^{\circ} \mathrm{C} / 12 \mathrm{PM}$ & $22.2^{\circ} \mathrm{C} / 6 \mathrm{AM}$ & $26.8^{\circ} \mathrm{C} / 12 \mathrm{PM}$ & $19.8^{\circ} \mathrm{C} / 6 \mathrm{AM}$ \\
\hline & 1.c. (3) & $28.1^{\circ} \mathrm{C} / 11 \mathrm{AM}$ & $22.7^{\circ} \mathrm{C} / 6 \mathrm{AM}$ & $27^{\circ} \mathrm{C} / 11 \mathrm{AM}$ & $19.4^{\circ} \mathrm{C} / 5 \mathrm{AM}$ \\
\hline & 1.d. (4) & $27.7^{\circ} \mathrm{C} / 3 \mathrm{PM}$ & $23.2^{\circ} \mathrm{C} / 5 \mathrm{AM}$ & $25.8^{\circ} \mathrm{C} / 3 \mathrm{PM}$ & $20.3^{\circ} \mathrm{C} / 8 \mathrm{AM}$ \\
\hline & 1.e. (4) & $27.3^{\circ} \mathrm{C} / 2 \mathrm{PM}$ & $22.8^{\circ} \mathrm{C} / 6 \mathrm{AM}$ & $24.8^{\circ} \mathrm{C} / 1 \mathrm{PM}$ & $20^{\circ} \mathrm{C} / 6 \mathrm{AM}$ \\
\hline \multirow{10}{*}{$\stackrel{\Xi ี \Xi}{\Sigma}$} & Indoor (1) & $26.4^{\circ} \mathrm{C} / 12 \mathrm{PM}$ & $23.5^{\circ} \mathrm{C} / 5: 30 \mathrm{AM}$ & $27.9^{\circ} \mathrm{C} / 4 \mathrm{PM}$ & $24.6^{\circ} \mathrm{C} / 5: 30 \mathrm{AM}$ \\
\hline & Outdoor (2) & $27.6^{\circ} \mathrm{C} / 12 \mathrm{PM}$ & $22.2^{\circ} \mathrm{C} / 6 \mathrm{AM}$ & $27^{\circ} \mathrm{C} / 12: 30 \mathrm{PM}$ & $19.8^{\circ} \mathrm{C} / 6 \mathrm{AM}$ \\
\hline & Outdoor (3) & $28.1^{\circ} \mathrm{C} / 11 \mathrm{AM}$ & $22.7^{\circ} \mathrm{C} / 6 \mathrm{AM}$ & $27^{\circ} \mathrm{C} / 11 \mathrm{AM}$ & $19.4^{\circ} \mathrm{C} / 5 \mathrm{AM}$ \\
\hline & Outdoor (4) & $27.5^{\circ} \mathrm{C} / 2: 30 \mathrm{PM}$ & $23^{\circ} \mathrm{C} / 5: 30 \mathrm{AM}$ & $25.3^{\circ} \mathrm{C} / 2 \mathrm{PM}$ & $20.2^{\circ} \mathrm{C} / 7 \mathrm{AM}$ \\
\hline & Difference (1)(2) & $0.6^{\circ} \mathrm{C}$ & $1.3^{\circ} \mathrm{C}$ & $0.9^{\circ} \mathrm{C}$ & $4.8^{\circ} \mathrm{C}$ \\
\hline & Difference (1)(3) & $1.5^{\circ} \mathrm{C}$ & $0.8^{\circ} \mathrm{C}$ & $0.9^{\circ} \mathrm{C}$ & $5.2^{\circ} \mathrm{C}$ \\
\hline & Difference (1)(4) & $0.9^{\circ} \mathrm{C}$ & $0.5^{\circ} \mathrm{C}$ & $2.6^{\circ} \mathrm{C}$ & $4.4^{\circ} \mathrm{C}$ \\
\hline & Time lag (1)(2) & 0 & $0.5 \mathrm{~h}$ & $3.5 \mathrm{~h}$ & 0 \\
\hline & Time lag (1)(3) & $1 \mathrm{~h}$ & $0.5 \mathrm{~h}$ & $5 \mathrm{~h}$ & $0.5 \mathrm{~h}$ \\
\hline & Time lag (1)(4) & 0 & 0 & $2 \mathrm{~h}$ & 0 \\
\hline
\end{tabular}

\section{B. Sasirangan Village}

Sasirangan village is a residential area of Seberang Mesjid located around the Martapura River. This area has long been a tourist destination in the city of Banjarmasin. In addition to being the center of the traditional clothing industry, this area also has a traditional house type that is a floating house. This floating house has a unique architectural building construction. The uniqueness of the floating house attract visitors to the Sasirangan Village and also becomes the center of the study of the Banjar traditional house, for academics and students (Figure 4).

The time lag between outdoor and indoor in floating houses is not happening. The outdoor thermal conditions greatly affect the indoor thermal conditions. The indoor thermal changes highly depend on the outdoor thermal environment. The mean of maximum dry bulb temperature is quite high from 2 PM to 3 PM. The mean of indoor maximum relative humidity is higher $9.4 \%$ than outdoor and quite high in the morning. The mean relative humidity is low during the day. The mean of indoor maximum wet bulb temperature is higher than the outdoor. The mean indoor maximum relative humidity is higher than outdoor as well as at minimum point. The relative humidity of open spaces is always higher than the relative humidity of indoor buildings. The maximum relative humidity difference between open space and building is about $7.5 \%$. The maximum relative humidity time lag is about 3 hours, except between indoor buildings with green open spaces only 0.5 hours. Maximum and minimum wet bulb temperature in open space is relatively the same. The effective temperature of green open space shows the best conditions, 
compared to the open space around floating market a very cold in the morning. The thermal and thermal comfort conditions in the floating houses can be seen in Table 4 .

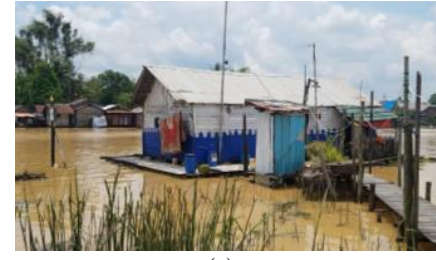

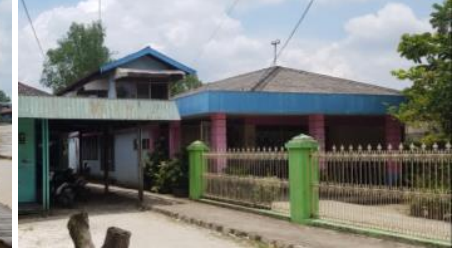

(b)
Fig 4. The environment condition in Sasirangan village, a) the floating house and b) houses in settlement

TABLE 4. Difference of Thermal Environment and Time Lag Measurement in Sasirangan Village

\begin{tabular}{|c|c|c|c|c|c|}
\hline \multirow{2}{*}{\multicolumn{2}{|c|}{$\begin{array}{c}\text { Measurment } \\
\text { Points }\end{array}$}} & \multicolumn{2}{|c|}{ Dry Bulb Temperature } & \multicolumn{2}{|c|}{ Relative Humidity } \\
\hline & & Max/time & Min/time & Max/time & Min/time \\
\hline \multicolumn{6}{|c|}{ Floating Houses } \\
\hline \multirow{4}{*}{ 2.a. } & Outdoor & $35.5^{\circ} \mathrm{C} / 2 \mathrm{PM}$ & $23.8^{\circ} \mathrm{C} / 5 \mathrm{AM}$ & $87.3 \% / 5 \mathrm{AM}$ & $50.6 \% / 1 \mathrm{PM}$ \\
\hline & Indoor & $36^{\circ} \mathrm{C} / 2 \mathrm{PM}$ & $24.3^{\circ} \mathrm{C} / 7 \mathrm{AM}$ & $86.9 \% / 5 \mathrm{AM}$ & $50.7 \% / 1 \mathrm{PM}$ \\
\hline & Difference & $0.5^{\circ} \mathrm{C}$ & $0.5^{\circ} \mathrm{C}$ & $0.4 \%$ & $0.1 \%$ \\
\hline & Time lag & 0 & $2 \mathrm{~h}$ & 0 & 0 \\
\hline \multirow{4}{*}{ 2.b. } & Outdoor & $34.7^{\circ} \mathrm{C} / 1 \mathrm{PM}$ & $23.5^{\circ} \mathrm{C} / 4 \mathrm{AM}$ & $87.8 \% / 5 \mathrm{AM}$ & $50.1 \% / 1 \mathrm{PM}$ \\
\hline & Indoor & $35.7^{\circ} \mathrm{C} / 1 \mathrm{PM}$ & $24.3^{\circ} \mathrm{C} / 4 \mathrm{AM}$ & $87.4 \% / 8 \mathrm{AM}$ & $49.1 \% / 1 \mathrm{PM}$ \\
\hline & Difference & $1^{\circ} \mathrm{C}$ & $0.8^{\circ} \mathrm{C}$ & $0.4 \%$ & $1 \%$ \\
\hline & Time lag & 0 & $\begin{array}{l}0 \\
\end{array}$ & $3 \mathrm{~h}$ & 0 \\
\hline \multirow{4}{*}{ 2.c. } & Outdoor & $36^{\circ} \mathrm{C} / 3 \mathrm{PM}$ & $24^{\circ} \mathrm{C} / 7 \mathrm{AM}$ & $89 \% / 6 \mathrm{AM}$ & $51.9 \% / 5 \mathrm{PM}$ \\
\hline & Indoor & $35.9^{\circ} \mathrm{C} / 3 \mathrm{PM}$ & $24.1^{\circ} \mathrm{C} / 7 \mathrm{AM}$ & $87.6 \% / 6 \mathrm{AM}$ & $52 \% / 5 \mathrm{PM}$ \\
\hline & Difference & $0.1^{\circ} \mathrm{C}$ & $0.1^{\circ} \mathrm{C}$ & $1.4 \%$ & $0.1 \%$ \\
\hline & Time lag & 0 & 0 & 0 & 0 \\
\hline \multirow{4}{*}{ 2.d. } & Outdoor & $37.4^{\circ} \mathrm{C} / 5 \mathrm{PM}$ & $24^{\circ} \mathrm{C} / 6 \mathrm{AM}$ & $87.5 \% / 7 \mathrm{AM}$ & $50.8 \% / 4 \mathrm{PM}$ \\
\hline & Indoor & $35.6^{\circ} \mathrm{C} / 4 \mathrm{PM}$ & $24^{\circ} \mathrm{C} / 6 \mathrm{AM}$ & $87.4 \% / 6 \mathrm{AM}$ & $52 \% / 4 \mathrm{PM}$ \\
\hline & Difference & $2^{\circ} \mathrm{C}$ & 0 & $0.1 \%$ & $1.20 \%$ \\
\hline & Time lag & 0 & 0 & 0 & 0 \\
\hline \multirow{4}{*}{ Mean } & Outdoor & $35.9^{\circ} \mathrm{C} / 3 \mathrm{PM}$ & $23.8^{\circ} \mathrm{C} / 6 \mathrm{AM}$ & $77.9 \% / 6 \mathrm{AM}$ & $50.9 \% / 2: 30 \mathrm{PM}$ \\
\hline & Indoor & $35.8^{\circ} \mathrm{C} / 2 \mathrm{PM}$ & $24.2^{\circ} \mathrm{C} / 6 \mathrm{AM}$ & $87.3 \% / 6 \mathrm{AM}$ & $51 \% / 2: 30 \mathrm{PM}$ \\
\hline & Difference & $0.1^{\circ} \mathrm{C}$ & $0.4^{\circ} \mathrm{C}$ & $9.4 \%$ & $0.1 \%$ \\
\hline & Time lag & 0 & 0 & 0 & 0 \\
\hline \multicolumn{6}{|c|}{ Houses in settlement } \\
\hline \multirow{4}{*}{ 2.e. } & Outdoor & $33.8^{\circ} \mathrm{C} / 3 \mathrm{PM}$ & $25.6^{\circ} \mathrm{C} / 7 \mathrm{AM}$ & $81 \% / 8 \mathrm{AM}$ & $57.3 \% / 3 \mathrm{PM}$ \\
\hline & Indoor & $32.9^{\circ} \mathrm{C} / 5 \mathrm{PM}$ & $27.6^{\circ} \mathrm{C} / 6 \mathrm{AM}$ & $78.8 \% / 10 \mathrm{AM}$ & $62.2 \% / 5 \mathrm{PM}$ \\
\hline & Difference & $0.9^{\circ} \mathrm{C}$ & $2^{\circ} \mathrm{C}$ & $2.2 \%$ & $4.90 \%$ \\
\hline & Time lag & 2 & 0 & 2 & 2 \\
\hline \multirow{4}{*}{ 2.f. } & Outdoor & $35^{\circ} \mathrm{C} / 3 \mathrm{PM}$ & $25.7^{\circ} \mathrm{C} / 5 \mathrm{AM}$ & $82.4 \% / 8 \mathrm{AM}$ & $55.8 \% / 3 \mathrm{PM}$ \\
\hline & Indoor & $32.9^{\circ} \mathrm{C} / 3 \mathrm{PM}$ & $27.6^{\circ} \mathrm{C} / 6 \mathrm{AM}$ & $80.4 \% / 9 \mathrm{AM}$ & $63 \% / 2 \mathrm{PM}$ \\
\hline & Difference & $2.1^{\circ} \mathrm{C}$ & $0.8^{\circ} \mathrm{C}$ & $2 \%$ & $7.20 \%$ \\
\hline & Time lag & 0 & $1 \mathrm{~h}$ & $2 \mathrm{~h}$ & 0 \\
\hline \multirow{4}{*}{ Mean } & Outdoor & $34.4^{\circ} \mathrm{C} / 3 \mathrm{PM}$ & $25.7^{\circ} \mathrm{C} / 6 \mathrm{AM}$ & $81.7 \% / 8 \mathrm{AM}$ & $59.8 \% / 3 \mathrm{PM}$ \\
\hline & Indoor & $32.9^{\circ} \mathrm{C} / 4 \mathrm{PM}$ & $27.6^{\circ} \mathrm{C} / 6 \mathrm{AM}$ & $79.6 \% / 9.30 \mathrm{AM}$ & $62.6 \% / 3: 30 \mathrm{PM}$ \\
\hline & Difference & $1.5^{\circ} \mathrm{C}$ & $1.9^{\circ} \mathrm{C}$ & $2.1 \%$ & $2.8 \%$ \\
\hline & Time lag & $1 \mathrm{~h}$ & 0 & $1.5 \mathrm{~h}$ & $0.5 \mathrm{~h}$ \\
\hline
\end{tabular}

The mean time lag occurs only in the maximum dry bulb temperature, maximum and minimum humidity. The mean of outdoor maximum dry bulb temperature is higher than the indoor maximum dry bulb temperature, as well as the mean of maximum outdoor relative humidity is higher than indoor. The mean of indoor wet bulb temperature first reaches the maximum and minimum points as well as the mean of the effective temperature (Table 5). In the sattlement, the indoor thermal comfort only in optimal comfort to warm comfort, but longer on uncomfortable conditions from day to night. In general, the outdoor thermal comfort conditions are always in the comfort zone, except for a moment in the morning and afternoon. The outdoor effective temperature is always lower than the indoor. The outdoor thermal comfort conditions are cooler than the indoor, especially from night to morning.
TABLE 5. Difference of Thermal Comfort and Time Lag Measurement in Sasirangan Village

\begin{tabular}{|c|c|c|c|c|c|}
\hline \multirow{2}{*}{\multicolumn{2}{|c|}{ Measurment Points }} & \multicolumn{2}{|c|}{ Wet Bulb Temperature } & \multicolumn{2}{|c|}{ Effective Temperature } \\
\hline & & Max/time & Min/time & Max/time & Min/time \\
\hline \multicolumn{6}{|c|}{ Floating Houses } \\
\hline \multirow{4}{*}{ 2.a. } & Outdoor & $27^{\circ} \mathrm{C} / 2 \mathrm{PM}$ & $22.2^{\circ} \mathrm{C} / 5 \mathrm{AM}$ & $26.9^{\circ} \mathrm{C} / 1 \mathrm{PM}$ & $20.5^{\circ} \mathrm{C} / 4 \mathrm{AM}$ \\
\hline & Indoor & $28.6^{\circ} \mathrm{C} / 12 \mathrm{PM}$ & $22.3^{\circ} \mathrm{C} / 2 \mathrm{AM}$ & $31.2^{\circ} \mathrm{C} / 5 \mathrm{PM}$ & $23.1^{\circ} \mathrm{C} / 4 \mathrm{AM}$ \\
\hline & Difference & $1.6^{\circ} \mathrm{C}$ & $0.1^{\circ} \mathrm{C}$ & $4.1^{\circ} \mathrm{C}$ & $2.6^{\circ} \mathrm{C}$ \\
\hline & Time lag & 0 & 0 & $4 \mathrm{~h}$ & 0 \\
\hline \multirow{4}{*}{ 2.b. } & Outdoor & $26.9^{\circ} \mathrm{C} / 12 \mathrm{PM}$ & $21.9^{\circ} \mathrm{C} / 4 \mathrm{AM}$ & $26.9^{\circ} \mathrm{C} / 12 \mathrm{PM}$ & $18.1^{\circ} \mathrm{C} / 4 \mathrm{AM}$ \\
\hline & Indoor & $28.3^{\circ} \mathrm{C} / 10 \mathrm{AM}$ & $22.4^{\circ} \mathrm{C} / 2 \mathrm{AM}$ & $30.4^{\circ} \mathrm{C} / 10 \mathrm{AM}$ & $23.8^{\circ} \mathrm{C} / 4 \mathrm{AM}$ \\
\hline & Difference & $1.2^{\circ} \mathrm{C}$ & $0.5^{\circ} \mathrm{C}$ & $3.5^{\circ} \mathrm{C}$ & $5.7^{\circ} \mathrm{C}$ \\
\hline & Time lag & 0 & 0 & 0 & 0 \\
\hline \multirow{4}{*}{ 2.c. } & Outdoor & $27.3^{\circ} \mathrm{C} / 3 \mathrm{PM}$ & $22.5^{\circ} \mathrm{C} / 7 \mathrm{AM}$ & $27.5^{\circ} \mathrm{C} / 3 \mathrm{PM}$ & $18.8^{\circ} \mathrm{C} / 7 \mathrm{AM}$ \\
\hline & Indoor & $28.3^{\circ} \mathrm{C} / 12 \mathrm{PM}$ & $22.5^{\circ} \mathrm{C} / 7 \mathrm{AM}$ & $29.7^{\circ} \mathrm{C} / 3 \mathrm{PM}$ & $22.9^{\circ} \mathrm{C} / 7 \mathrm{AM}$ \\
\hline & Difference & $1^{\circ} \mathrm{C}$ & 0 & $2.2^{\circ} \mathrm{C}$ & $4.1^{\circ} \mathrm{C}$ \\
\hline & Time lag & 0 & 0 & 0 & 0 \\
\hline \multirow{4}{*}{ 2.d. } & Outdoor & $28.7^{\circ} \mathrm{C} / 5 \mathrm{PM}$ & $22.4^{\circ} \mathrm{C} / 6 \mathrm{AM}$ & $29.8^{\circ} \mathrm{C} / 5 \mathrm{PM}$ & $18.9^{\circ} \mathrm{C} / 7 \mathrm{AM}$ \\
\hline & Indoor & $27.8^{\circ} \mathrm{C} / 1 \mathrm{PM}$ & $22.4^{\circ} \mathrm{C} / 6 \mathrm{AM}$ & $29.8^{\circ} \mathrm{C} / 1 \mathrm{PM}$ & $22.8^{\circ} \mathrm{C} / 6 \mathrm{AM}$ \\
\hline & Difference & $0.9^{\circ} \mathrm{C}$ & 0 & 0 & $3.9^{\circ} \mathrm{C}$ \\
\hline & Time lag & 0 & 0 & 0 & 0 \\
\hline \multirow{4}{*}{ Mean } & Outdoor & $27.5^{\circ} \mathrm{C} / 2: 30 \mathrm{PM}$ & $22.3^{\circ} \mathrm{C} / 6 \mathrm{AM}$ & $27.8^{\circ} \mathrm{C} / 2: 30 \mathrm{PM}$ & $19.1^{\circ} \mathrm{C} / 5: 30 \mathrm{AM}$ \\
\hline & Indoor & $28.3^{\circ} \mathrm{C} / 12 \mathrm{PM}$ & $22.4^{\circ} \mathrm{C} / 4 \mathrm{AM}$ & $30.3^{\circ} \mathrm{C} / 12: 30 \mathrm{PM}$ & $23.2^{\circ} \mathrm{C} / 5: 30 \mathrm{AM}$ \\
\hline & Difference & $0.8^{\circ} \mathrm{C}$ & $0.1^{\circ} \mathrm{C}$ & $2.5^{\circ} \mathrm{C}$ & $4.1^{\circ} \mathrm{C}$ \\
\hline & Time lag & 0 & 0 & 0 & 0 \\
\hline \multicolumn{6}{|c|}{ Houses in settlements } \\
\hline \multirow{4}{*}{ 2.e. } & Outdoor & $28.3^{\circ} \mathrm{C} / 1 \mathrm{PM}$ & $22.3^{\circ} \mathrm{C} / 5 \mathrm{AM}$ & $27.1^{\circ} \mathrm{C} / 1 \mathrm{PM}$ & $19.8^{\circ} \mathrm{C} / 7 \mathrm{AM}$ \\
\hline & Indoor & $28^{\circ} \mathrm{C} / 12 \mathrm{PM}$ & $23.5^{\circ} \mathrm{C} / 5 \mathrm{AM}$ & $28.9^{\circ} \mathrm{C} / 10 \mathrm{AM}$ & $24.7^{\circ} \mathrm{C} / 6 \mathrm{AM}$ \\
\hline & Difference & $0.3^{\circ} \mathrm{C}$ & $1.2^{\circ} \mathrm{C}$ & $2.8^{\circ} \mathrm{C}$ & $5.5^{\circ} \mathrm{C}$ \\
\hline & Time lag & 0 & 0 & 0 & 0 \\
\hline \multirow{4}{*}{ 2.f. } & Outdoor & $28.1^{\circ} \mathrm{C} / 12 \mathrm{PM}$ & $22.7^{\circ} \mathrm{C} / 5 \mathrm{AM}$ & $27.2^{\circ} \mathrm{C} / 2 \mathrm{PM}$ & $20.1^{\circ} \mathrm{C} / 5 \mathrm{AM}$ \\
\hline & Indoor & $26.8^{\circ} \mathrm{C} / 11 \mathrm{AM}$ & $23.8^{\circ} \mathrm{C} / 5 \mathrm{AM}$ & $28.3^{\circ} \mathrm{C} / 4 \mathrm{PM}$ & $24.4^{\circ} \mathrm{C} / 5 \mathrm{AM}$ \\
\hline & Difference & $1.3^{\circ} \mathrm{C}$ & $1.1^{\circ} \mathrm{C}$ & $1.1^{\circ} \mathrm{C}$ & $4.3^{\circ} \mathrm{C}$ \\
\hline & Time lag & 0 & 0 & 2 & 0 \\
\hline \multirow{4}{*}{ Mean } & Outdoor & $28.2^{\circ} \mathrm{C} / 12: 30 \mathrm{PM}$ & $22.5^{\circ} \mathrm{C} / 5 \mathrm{AM}$ & $27.2^{\circ} \mathrm{C} / 1.30 \mathrm{PM}$ & $20^{\circ} \mathrm{C} / 6 \mathrm{AM}$ \\
\hline & Indoor & $27.4^{\circ} \mathrm{C} / 11: 30 \mathrm{AM}$ & $23.7^{\circ} \mathrm{C} / 5 \mathrm{AM}$ & $28.6^{\circ} \mathrm{C} / 1 \mathrm{PM}$ & $24.6^{\circ} \mathrm{C} / 5: 30 \mathrm{AM}$ \\
\hline & Difference & $0.8^{\circ} \mathrm{C}$ & $1.2^{\circ} \mathrm{C}$ & $2.5^{\circ} \mathrm{C}$ & $4.6^{\circ} \mathrm{C}$ \\
\hline & Time lag & 0 & 0 & 0 & 0 \\
\hline
\end{tabular}

\section{Bilu Village}

Bilu Village is a traditional settlement in Banjarmasin City. This village includes the old town whose people's activity are highly dependent on the Martapura River. This is evident from the orientation of the house facing the river. Along the banks of the river there is a pedestrian way. This pedestrian way has been around since the beginning of the settlement was built. Pedestrian way in Bilu village is also used by residents as a place of social interaction, so this pedestrian path also becomes a public space area. Since 2016 , this settlement has been arranged by the City Government. The pedestrian way is being renovated and the river-oriented houses are painted in green to make it look attractive, clean and no more slums (Figure 5).

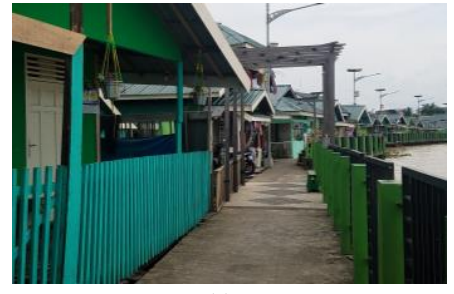

(a)

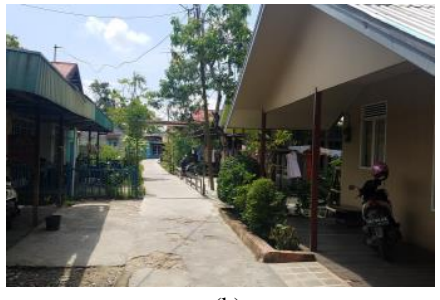

Fig 5. The environment condition in Bilu village, a) the houses aside pedestrian way and $b$ ) houses in settlement

The thermal environment conditions in Bilu village can be seen in Table 6. The mean maximum dry bulb temperature is quite high and indoor and outdoor conditions are relatively not much different. The mean value of maximum relative humidity is also quite high, and the minimum value is quite low. The mean of maximum wet bulb temperature in indoor of houses aside pedestrian way are higher than outdoors, as well as the mean of minimum wet bulb temperature. The mean of 
maximum effective temperature in indoor of houses aside pedestrian ways are is higher than the outdoor with a difference of $2.7^{\circ} \mathrm{C}$ ET. On the other hand, the mean of minimum effective temperature, outdoor conditions are lower than the indoor by a difference of $4.7^{\circ} \mathrm{C}$ ET (Table 7 ). These conditions indicate the indoor conditions are hotter when the effective temperature is maximized, and cooler outdoor conditions when effective temperature is minimized.

TABLE 6. Difference of Thermal Environment and Time Lag Measurement in Bilu Village

\begin{tabular}{|c|c|c|c|c|c|}
\hline \multirow{2}{*}{\multicolumn{2}{|c|}{ Measurment ponts }} & \multicolumn{2}{|c|}{ Dry Bulb Temperature } & \multicolumn{2}{|c|}{ Relative Humidity } \\
\hline & & Max/time & Min/time & Max/time & Min/time \\
\hline \multicolumn{6}{|c|}{ Houses aside pedestrian way } \\
\hline \multirow{4}{*}{ 3.a. } & Outdoor & $37.3^{\circ} \mathrm{C} / 2 \mathrm{PM}$ & $24.8^{\circ} \mathrm{C} / 5 \mathrm{AM}$ & $86.3 \% / 7 \mathrm{AM}$ & $41.5 \% / 2 \mathrm{PM}$ \\
\hline & Indoor & $37.2^{\circ} \mathrm{C} / 2 \mathrm{PM}$ & $25.4^{\circ} \mathrm{C} / 7 \mathrm{AM}$ & $83.8 \% / 8 \mathrm{AM}$ & $45.8 \% / 2 \mathrm{PM}$ \\
\hline & Difference & $0.1^{\circ} \mathrm{C}$ & $0.6^{\circ} \mathrm{C}$ & $2.5 \%$ & $5.30 \%$ \\
\hline & Time lag & 0 & $2 \mathrm{~h}$ & $1 \mathrm{~h}$ & 0 \\
\hline \multirow{4}{*}{ 3.b. } & Outdoor & $36.1^{\circ} \mathrm{C} / 1 \mathrm{PM}$ & $24.4^{\circ} \mathrm{C} / 6 \mathrm{AM}$ & $86.6 \% / 7 \mathrm{AM}$ & $42.5 \% / 2 \mathrm{PM}$ \\
\hline & Indoor & $36.7^{\circ} \mathrm{C} / 2 \mathrm{PM}$ & $25.2^{\circ} \mathrm{C} / 6 \mathrm{AM}$ & $84.8 \% / 7 \mathrm{AM}$ & $47.4 \% / 2 \mathrm{PM}$ \\
\hline & Difference & $0.6^{\circ} \mathrm{C}$ & $0.8^{\circ} \mathrm{C}$ & $1.80 \%$ & $4.90 \%$ \\
\hline & Time lag & 0 & 0 & $3 \mathrm{~h}$ & 0 \\
\hline \multirow{4}{*}{ 3.c. } & Outdoor & $36.3^{\circ} \mathrm{C} / 2 \mathrm{PM}$ & $23.3^{\circ} \mathrm{C} / 6 \mathrm{AM}$ & $89.8 \% / 8 \mathrm{AM}$ & $45.8 \% / 3 \mathrm{PM}$ \\
\hline & Indoor & $36^{\circ} \mathrm{C} / 2 \mathrm{PM}$ & $24.2^{\circ} \mathrm{C} / 6 \mathrm{AM}$ & $87.4 \% / 8 \mathrm{AM}$ & $50.5 \% / 2 \mathrm{PM}$ \\
\hline & Difference & $0.3^{\circ} \mathrm{C}$ & $0.9^{\circ} \mathrm{C}$ & $2.40 \%$ & $4.70 \%$ \\
\hline & Time lag & 0 & 0 & 0 & 0 \\
\hline \multirow{4}{*}{ 3.d. } & Outdoor & $36.2^{\circ} \mathrm{C} / 1 \mathrm{PM}$ & $23.5^{\circ} \mathrm{C} / 7 \mathrm{AM}$ & $88 \% / 7 \mathrm{AM}$ & $44.8 \% / 2 \mathrm{PM}$ \\
\hline & Indoor & $35.4^{\circ} \mathrm{C} / 3 \mathrm{PM}$ & $24.2^{\circ} \mathrm{C} / 7 \mathrm{AM}$ & $87.3 \% / 7 \mathrm{AM}$ & $52.1 \% / 3 \mathrm{PM}$ \\
\hline & Difference & $0.8^{\circ} \mathrm{C}$ & $0.7^{\circ} \mathrm{C}$ & $0.30 \%$ & $7.30 \%$ \\
\hline & Time lag & $2 \mathrm{~h}$ & 0 & 0 & $1 \mathrm{~h}$ \\
\hline \multirow{4}{*}{ Mean } & Outdoor & $36.5^{\circ} \mathrm{C} / 1.30 \mathrm{PM}$ & $24^{\circ} \mathrm{C} / 6 \mathrm{AM}$ & $87.7 \% / 7 \mathrm{AM}$ & $43.7 \% / 2 \mathrm{PM}$ \\
\hline & Indoor & $36.3^{\circ} \mathrm{C} / 2 \mathrm{PM}$ & $24.8^{\circ} \mathrm{C} / 6: 30 \mathrm{AM}$ & $85.8 \% / 7: 30 \mathrm{AM}$ & $49 \% / 2: 30 \mathrm{PM}$ \\
\hline & Difference & $0.2^{\circ} \mathrm{C}$ & $0.8^{\circ} \mathrm{C}$ & $1.9 \%$ & $5.30 \%$ \\
\hline & Time lag & $0.5 \mathrm{~h}$ & $0.5 \mathrm{~h}$ & $0.5 \mathrm{~h}$ & $0.5 \mathrm{~h}$ \\
\hline \multicolumn{6}{|c|}{ Houses in settlement } \\
\hline \multirow{4}{*}{ 3.e. } & Outdoor & $32.4^{\circ} \mathrm{C} / 12 \mathrm{PM}$ & $24.2^{\circ} \mathrm{C} / 4 \mathrm{AM}$ & $83.5 \% / 6 \mathrm{AM}$ & $69.2 \% / 2 \mathrm{PM}$ \\
\hline & Indoor & $29.9^{\circ} \mathrm{C} / 1 \mathrm{PM}$ & $26.7^{\circ} \mathrm{C} / 7 \mathrm{AM}$ & $77.9 \% / 7 \mathrm{AM}$ & $71.6 \% / 4 \mathrm{PM}$ \\
\hline & Difference & $2.5^{\circ} \mathrm{C}$ & $1,1^{\circ} \mathrm{C}$ & $5.60 \%$ & $2.40 \%$ \\
\hline & Time lag & 1 & 3 & 1 & 2 \\
\hline \multirow{4}{*}{ 3.f. } & Outdoor & $33.7^{\circ} \mathrm{C} / 1 \mathrm{PM}$ & $24.7^{\circ} \mathrm{C} / 5 \mathrm{AM}$ & $83 \% / 7 \mathrm{AM}$ & $66.4 \% / 3 \mathrm{PM}$ \\
\hline & Indoor & $31.7^{\circ} \mathrm{C} / 1 \mathrm{PM}$ & $26.5^{\circ} \mathrm{C} / 5 \mathrm{AM}$ & $81.6 \% / 9 \mathrm{AM}$ & $71 \% / 2 \mathrm{PM}$ \\
\hline & Difference & $2^{\circ} \mathrm{C}$ & $1.2^{\circ} \mathrm{C}$ & $1.40 \%$ & $5.40 \%$ \\
\hline & Time lag & 0 & 0 & $2 \mathrm{~h}$ & 0 \\
\hline \multirow{4}{*}{ Mean } & Outdoor & $33.1^{\circ} \mathrm{C} / 12.30 \mathrm{PM}$ & $24.5^{\circ} \mathrm{C} / 4.30 \mathrm{AM}$ & $83.3 \% / 6: 30 \mathrm{AM}$ & $67.8 \% / 2: 30 \mathrm{PM}$ \\
\hline & Indoor & $30.8^{\circ} \mathrm{C} / 1 \mathrm{PM}$ & $26.6^{\circ} \mathrm{C} / 6 \mathrm{AM}$ & $79.8 \% / 8 \mathrm{AM}$ & $71.3 \% / 3 \mathrm{PM}$ \\
\hline & Difference & $2.9^{\circ} \mathrm{C}$ & $2.1^{\circ} \mathrm{C}$ & $3.5 \%$ & $3.5 \%$ \\
\hline & Time lag & $0.5 \mathrm{~h}$ & $1.5 \mathrm{~h}$ & $1.5 \mathrm{~h}$ & $0.5 \mathrm{~h}$ \\
\hline
\end{tabular}

Each house has a time lag of about 0.5 hours to 1.5 hours, but at the mean of wet bulb temperature and minimum effective temperature there is no time lag. In the settlement houses, the mean of maximum dry bulb temperature in outdoor is higher than indoor with a difference of $1.2^{\circ} \mathrm{C}$. On the other hand, the mean of minimum dry bulb temperature in outdoor is lower. The mean of maximum relative humidity in outdoor is higher. The mean of maximum wet bulb temperature in outdoor is higher than indoor. The mean of maximum effective temperature in indoor is higher than the outdoor with a difference of $1.7^{\circ} \mathrm{C}$ ET. On the other hand, the mean of minimum effective temperature in outdoor conditions are lower than indoor by a margin of $5.6^{\circ} \mathrm{C}$ ET (Table 7). These conditions indicate the thermal comfort conditions between the houses aside pedestrian ways and the houses in settlement is same. The indoor conditions are hotter when the effective temperature is maximized, and cooler outdoor conditions when effective temperature is minimized.
TABLE 7. Difference of Thermal Comfort and Time Lag Measurement in Sasirangan Village

\begin{tabular}{|c|c|c|c|c|c|}
\hline \multirow{2}{*}{\multicolumn{2}{|c|}{ Measurment ponts }} & \multicolumn{2}{|c|}{ Dry Bulb Temperature } & \multicolumn{2}{|c|}{ Relative Humidity } \\
\hline & & Max/time & Min/time & Max/time & Min/time \\
\hline \multicolumn{6}{|c|}{ Houses aside pedestrian way } \\
\hline \multirow{4}{*}{ 3.a. } & Outdoor & $26.4^{\circ} \mathrm{C} / 12 \mathrm{PM}$ & $22.9^{\circ} \mathrm{C} / 6 \mathrm{AM}$ & $27.3^{\circ} \mathrm{C} / 2 \mathrm{PM}$ & $19.8^{\circ} \mathrm{C} / 6 \mathrm{AM}$ \\
\hline & Indoor & $27.7^{\circ} \mathrm{C} / 12 \mathrm{PM}$ & $23^{\circ} \mathrm{C} / 6 \mathrm{AM}$ & $30.1^{\circ} \mathrm{C} / 2 \mathrm{PM}$ & $23.9^{\circ} \mathrm{C} / 6 \mathrm{AM}$ \\
\hline & Difference & $1.3^{\circ} \mathrm{C}$ & $0.1^{\circ} \mathrm{C}$ & $2.8^{\circ} \mathrm{C}$ & $4.1^{\circ} \mathrm{C}$ \\
\hline & Time lag & 0 & 0 & 0 & 0 \\
\hline \multirow{4}{*}{ 3.b. } & Outdoor & $26^{\circ} \mathrm{C} / 9 \mathrm{AM}$ & $22.6^{\circ} \mathrm{C} / 6 \mathrm{AM}$ & $27^{\circ} \mathrm{C} / 3 \mathrm{PM}$ & $19.1^{\circ} \mathrm{C} / 6 \mathrm{AM}$ \\
\hline & Indoor & $27.4^{\circ} \mathrm{C} / 10 \mathrm{AM}$ & $22.9^{\circ} \mathrm{C} / 6 \mathrm{AM}$ & $29.5^{\circ} \mathrm{C} / 1 \mathrm{PM}$ & $23.3^{\circ} \mathrm{C} / 6 \mathrm{AM}$ \\
\hline & Difference & $1.4^{\circ} \mathrm{C}$ & $0.3^{\circ} \mathrm{C}$ & $2.5^{\circ} \mathrm{C}$ & $4.2^{\circ} \mathrm{C}$ \\
\hline & Time lag & 0 & 0 & 0 & 0 \\
\hline \multirow{4}{*}{ 3.c. } & Outdoor & $26.7^{\circ} \mathrm{C} / 2 \mathrm{PM}$ & $21.8^{\circ} \mathrm{C} / 6 \mathrm{AM}$ & $27.7^{\circ} \mathrm{C} / 2 \mathrm{PM}$ & $17.8^{\circ} \mathrm{C} / 7 \mathrm{AM}$ \\
\hline & Indoor & $27.3^{\circ} \mathrm{C} / 1 \mathrm{PM}$ & $22.3^{\circ} \mathrm{C} / 6 \mathrm{AM}$ & $29.8^{\circ} \mathrm{C} / 2 \mathrm{PM}$ & $23^{\circ} \mathrm{C} / 5 \mathrm{AM}$ \\
\hline & Difference & $0.6^{\circ} \mathrm{C}$ & $0.5^{\circ} \mathrm{C}$ & $2^{\circ} \mathrm{C}$ & $5.2^{\circ} \mathrm{C}$ \\
\hline & Time lag & 0 & 0 & 0 & 0 \\
\hline \multirow{4}{*}{ 3.d. } & Outdoor & $26.1^{\circ} \mathrm{C} / 1 \mathrm{PM}$ & $21.8^{\circ} \mathrm{C} / 4 \mathrm{AM}$ & $26.9^{\circ} \mathrm{C} / 1 \mathrm{PM}$ & $18^{\circ} \mathrm{C} / 7 \mathrm{AM}$ \\
\hline & Indoor & $27.7^{\circ} \mathrm{C} / 1 \mathrm{PM}$ & $22.3^{\circ} \mathrm{C} / 5 \mathrm{AM}$ & $30^{\circ} \mathrm{C} / 1 \mathrm{PM}$ & $23.2^{\circ} \mathrm{C} / 5 \mathrm{AM}$ \\
\hline & Difference & $1.6^{\circ} \mathrm{C}$ & $0.5^{\circ} \mathrm{C}$ & $3.1^{\circ} \mathrm{C}$ & $5.2^{\circ} \mathrm{C}$ \\
\hline & Time lag & 0 & $1 \mathrm{~h}$ & 0 & 0 \\
\hline \multirow{4}{*}{ Mean } & Outdoor & $26.3^{\circ} \mathrm{C} / 11.30 \mathrm{AM}$ & $22.3^{\circ} \mathrm{C} / 5: 30 \mathrm{AM}$ & $27.2^{\circ} \mathrm{C} / 2 \mathrm{PM}$ & $18.7^{\circ} \mathrm{C} / 6: 30 \mathrm{AM}$ \\
\hline & Indoor & $27.5^{\circ} \mathrm{C} / 12 \mathrm{PM}$ & $22.7^{\circ} \mathrm{C} / 6 \mathrm{AM}$ & $29.9^{\circ} \mathrm{C} / 1: 30 \mathrm{PM}$ & $23.4^{\circ} \mathrm{C} / 5: 30 \mathrm{AM}$ \\
\hline & Difference & $1.2^{\circ} \mathrm{C}$ & $0.4^{\circ} \mathrm{C}$ & $2.7^{\circ} \mathrm{C}$ & $4.7^{\circ} \mathrm{C}$ \\
\hline & Time lag & $0.5 \mathrm{~h}$ & $0.5 \mathrm{~h}$ & 0 & 0 \\
\hline \multicolumn{6}{|c|}{ Houses in settlement } \\
\hline \multirow{4}{*}{ 3.e. } & Outdoor & $27.5^{\circ} \mathrm{C} / 12 \mathrm{PM}$ & $22.3^{\circ} \mathrm{C} / 6 \mathrm{AM}$ & $24.8^{\circ} \mathrm{C} / 12 \mathrm{PM}$ & $19^{\circ} \mathrm{C} / 4 \mathrm{AM}$ \\
\hline & Indoor & $25.9^{\circ} \mathrm{C} / 1 \mathrm{PM}$ & $23.6^{\circ} \mathrm{C} / 6 \mathrm{AM}$ & $27.1^{\circ} \mathrm{C} / 12 \mathrm{PM}$ & $24.8^{\circ} \mathrm{C} / 6 \mathrm{AM}$ \\
\hline & Difference & $1.6^{\circ} \mathrm{C}$ & $1.3^{\circ} \mathrm{C}$ & $2.3^{\circ} \mathrm{C}$ & $5.8^{\circ} \mathrm{C}$ \\
\hline & Time lag & 1 & 0 & 0 & 2 \\
\hline \multirow{4}{*}{ 3.f. } & Outdoor & $29.1^{\circ} \mathrm{C} / 1 \mathrm{PM}$ & $22.2^{\circ} \mathrm{C} / 5 \mathrm{AM}$ & $28^{\circ} \mathrm{C} / 1 \mathrm{PM}$ & $19^{\circ} \mathrm{C} / 5 \mathrm{AM}$ \\
\hline & Indoor & $28.2^{\circ} \mathrm{C} / 12 \mathrm{PM}$ & $23.2^{\circ} \mathrm{C} / 5 \mathrm{AM}$ & $29.1^{\circ} \mathrm{C} / 2 \mathrm{PM}$ & $24.1^{\circ} \mathrm{C} / 3 \mathrm{AM}$ \\
\hline & Difference & $0.9^{\circ} \mathrm{C}$ & $1^{\circ} \mathrm{C}$ & $1.1^{\circ} \mathrm{C}$ & $5.1^{\circ} \mathrm{C}$ \\
\hline & Time lag & $\begin{array}{l}0 \\
\end{array}$ & 0 & 1 & 0 \\
\hline \multirow{4}{*}{ Mean } & Outdoor & $28.3^{\circ} \mathrm{C} / 12: 30 \mathrm{PM}$ & $22.3^{\circ} \mathrm{C} / 5: 30 \mathrm{AM}$ & $26.4^{\circ} \mathrm{C} / 12: 30 \mathrm{PM}$ & $19^{\circ} \mathrm{C} / 4: 30 \mathrm{AM}$ \\
\hline & Indoor & $27.1^{\circ} \mathrm{C} / 12: 30 \mathrm{AM}$ & $23.4^{\circ} \mathrm{C} / 5: 30 \mathrm{AM}$ & $28.1^{\circ} \mathrm{C} / 1 \mathrm{PM}$ & $24.6^{\circ} \mathrm{C} / 4: 30 \mathrm{AM}$ \\
\hline & Difference & $1.2^{\circ} \mathrm{C}$ & $1.1^{\circ} \mathrm{C}$ & $1.7^{\circ} \mathrm{C}$ & $5.6^{\circ} \mathrm{C}$ \\
\hline & Time lag & & & $0.5 \mathrm{~h}$ & \\
\hline
\end{tabular}

\section{RESEARCH RESULT}

Indoor effective temperatures always exceeded the threshold of thermal comfort SNI 03-6572. The value of the effective temperature was higher in floating houses in Sasirangan village and houses aside pedestrian ways in Bilu village. The highest effective temperature in both places this happened during the day, about $30^{\circ} \mathrm{C}$ ET. The outdoor effective temperature down just past the threshold of thermal comfort SNI 03-6572, as shown in Table 5 and Table 7. Almost all places had minimum effective temperatures outdoor around $20^{\circ} \mathrm{C} \mathrm{ET}$ and occurred in the morning.

These conditions indicate that the environmental conditions outdoor relatively comfortable to move. Green open space is the best condition. Effective temperature is always in the comfort zone. This proves the more open green space, the better micro-climatic conditions. Effective temperatures around the river are lower than the mainland region. The difference of effective temperature between the river and the mainland is $0.5^{\circ} \mathrm{C}$ ET- $0.9^{\circ} \mathrm{C} \mathrm{ET}$, as shown in Table 9. The difference of minimum effective temperature between indoor and outdoor is highest about $3.88^{\circ} \mathrm{C}$ ET to $5.15^{\circ} \mathrm{C}$ ET. The difference of the mean effective temperature in floating house is highest about $4.30^{\circ} \mathrm{C}$ ET.

If it is correlated between the maximum and minimum temperature with time, it is known that the indoor minimum effective temperature occurs from 4:30 AM to 6 AM while outdoor from 4:30 AM to $7 \mathrm{AM}$. In addition, the maximum effective temperatures in indoor is about from 12:30 AM to 1:30 AM, except for indoor buildings in green open spaces located: slower to reach maximum point and maximum value lower than building or house in Sasirangan and Bilu village. On the other hand, the outdoor maximum effective temperature conditions from $12.30 \mathrm{PM}$ to $2.30 \mathrm{PM}$ and the 
effective temperature in open space first reach the maximum point at 11 AM. As shown in Table 8.

TABLE 8. Difference of Mean Effective Temperature in Research Places

\begin{tabular}{|c|c|c|c|}
\hline \multirow{2}{*}{\multicolumn{2}{|c|}{ Research Places }} & \multicolumn{2}{|c|}{ Mean Effective Temperature } \\
\hline & & Max/time & Min/time \\
\hline \multicolumn{4}{|l|}{ Siring Tendean } \\
\hline \multirow{4}{*}{ Building } & Outdoor & $27^{\circ} \mathrm{C} / 12: 30 \mathrm{PM}$ & $19.8^{\circ} \mathrm{C} / 6 \mathrm{AM}$ \\
\hline & Indoor & $27.9^{\circ} \mathrm{C} / 4 \mathrm{PM}$ & $24.6^{\circ} \mathrm{C} / 5: 30 \mathrm{AM}$ \\
\hline & Difference & $0.9^{\circ} \mathrm{C}$ & $4.8^{\circ} \mathrm{C}$ \\
\hline & Time lag & $3.5 \mathrm{~h}$ & 0 \\
\hline \multirow[t]{2}{*}{ Open Space } & $\begin{array}{c}\text { Floating } \\
\text { Market }\end{array}$ & $27^{\circ} \mathrm{C} / 11 \mathrm{AM}$ & $19.4^{\circ} \mathrm{C} / 5 \mathrm{AM}$ \\
\hline & Green OS & $25.3^{\circ} \mathrm{C} / 2 \mathrm{PM}$ & $20.2^{\circ} \mathrm{C} / 7 \mathrm{AM}$ \\
\hline \multicolumn{4}{|l|}{ Sasirangan Village } \\
\hline \multirow{4}{*}{ Floating House } & Outdoor & $27.8^{\circ} \mathrm{C} / 2: 30 \mathrm{PM}$ & $19.1^{\circ} \mathrm{C} / 5: 30 \mathrm{AM}$ \\
\hline & Indoor & $30.3^{\circ} \mathrm{C} / 12: 30 \mathrm{PM}$ & $23.2^{\circ} \mathrm{C} / 5: 30 \mathrm{AM}$ \\
\hline & Difference & $2,5^{\circ} \mathrm{C}$ & $4.1^{\circ} \mathrm{C}$ \\
\hline & Time lag & 0 & 0 \\
\hline \multirow{4}{*}{ Settlement } & Outdoor & $27.2^{\circ} \mathrm{C} / 1: 30 \mathrm{PM}$ & $20^{\circ} \mathrm{C} / 6 \mathrm{AM}$ \\
\hline & Indoor & $28.6^{\circ} \mathrm{C} / 1 \mathrm{PM}$ & $24.6^{\circ} \mathrm{C} / 5: 30 \mathrm{AM}$ \\
\hline & Difference & $2.5^{\circ} \mathrm{C}$ & $4.6^{\circ} \mathrm{C}$ \\
\hline & Time lag & 0 & 0 \\
\hline \multicolumn{4}{|l|}{ Bilu Village } \\
\hline \multirow{4}{*}{$\begin{array}{c}\text { House aside } \\
\text { pedestrian ways }\end{array}$} & Outdoor & $27.2^{\circ} \mathrm{C} / 2 \mathrm{PM}$ & $18.7^{\circ} \mathrm{C} / 6: 30 \mathrm{AM}$ \\
\hline & Indoor & $29.9^{\circ} \mathrm{C} / 1: 30 \mathrm{PM}$ & $23.4^{\circ} \mathrm{C} / 5: 30 \mathrm{AM}$ \\
\hline & Difference & $2.7^{\circ} \mathrm{C}$ & $4.7^{\circ} \mathrm{C}$ \\
\hline & Time lag & 0 & 0 \\
\hline \multirow{4}{*}{ Settlement } & Outdoor & $26.4^{\circ} \mathrm{C} / 12: 30 \mathrm{PM}$ & $19^{\circ} \mathrm{C} / 4: 30 \mathrm{AM}$ \\
\hline & Indoor & $28.1^{\circ} \mathrm{C} / 1 \mathrm{PM}$ & $24.6^{\circ} \mathrm{C} / 4: 30 \mathrm{AM}$ \\
\hline & Difference & $1.7^{\circ} \mathrm{C}$ & $5.6^{\circ} \mathrm{C}$ \\
\hline & Time lag & $0,5 \mathrm{~h}$ & 0 \\
\hline
\end{tabular}

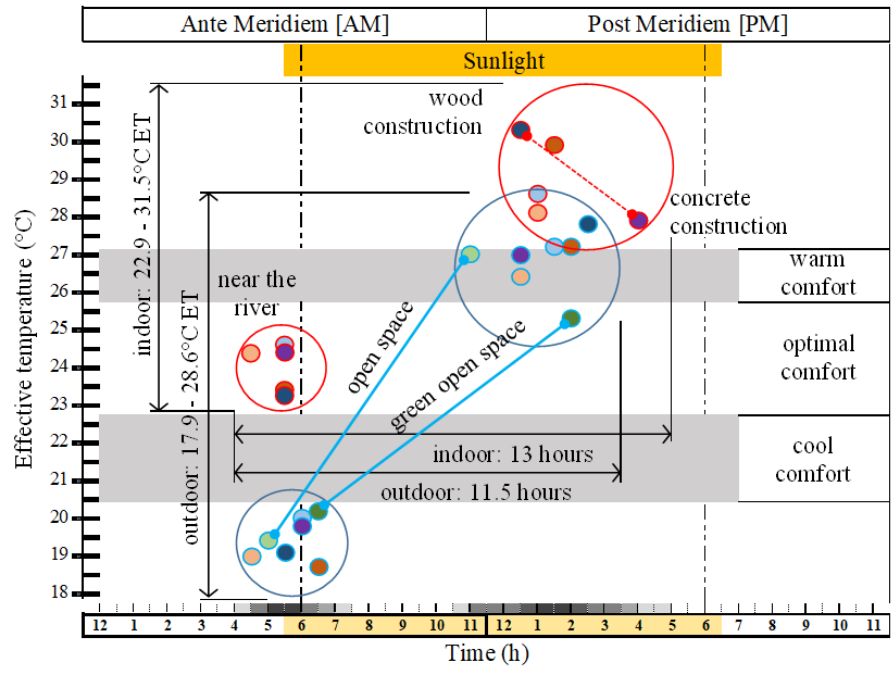

Fig 6. The characteristics of mean effective temperature in research places

The mean of outdoor minimum effective temperature is lower between $2.5^{\circ} \mathrm{C}$ ET to $5^{\circ} \mathrm{C}$ ET, compared to indoor conditions. Likewise, the mean of maximum effective temperature in outdoor is lower between $0^{\circ} \mathrm{C} \mathrm{ET}$ to $5^{\circ} \mathrm{C} \mathrm{ET}$. The range of outdoor effective temperature between minimum and maximum is about $11^{\mathrm{O}} \mathrm{C}$ ET and from minimum to maximum value takes about 11 hours and 30 minutes. On the other hand, the range of indoor effective temperature between the minimum and maximum value of about $8.5^{\circ} \mathrm{C}$ ET and from the minimum to maximum value takes about 13 hours. The conditions of outdoor minimum temperature are in the uncomfortable zone below $20.5^{\circ} \mathrm{C}$ ET, while the indoor minimum effective temperature is in the optimum comfortable zone between $23^{\circ} \mathrm{C}$ ET to $25^{\circ} \mathrm{C}$ ET. At a maximum effective temperature of outdoor conditions are in the optimum zone, warm and in an uncomfortable zone ebove $27.1^{\circ} \mathrm{C}$ ET. The indoor maximum effective temperature is always in the uncomfortable zone. The condition of the building with wooden construction is higher the effective temperature compared with concrete construction buildings, because the wooden building with roof material is zinc. The wood construction reaches maximum value faster than the structure concrete (Figure 6). The green open space conditions have the best effective temperature compared to the others. The condition of effective temperatures of green open space has been always in the comfort zone SNI 03-6572. On the other hand, open space without vegetation at minimum conditions in uncomfortable zone, and effective temperature reached maximum point, faster than green open space.

After the text edit has been completed, the paper is ready for the template. Duplicate the template file by using the Save As command, and use the naming convention prescribed by your conference for the name of your paper. In this newly created file, highlight all of the contents and import your prepared text file. You are now ready to style your paper; use the scroll down window on the left of the MS Word Formatting toolbar.

\section{DISCUSSION: THERMAL COMFORT EVALUATION}

In all study sites, the outdoor thermal environment conditions were lower and always on the comfort zone during the day. The outdoor effective temperatures never exceeded $27.1^{\circ} \mathrm{C}$ ET. The outdoor thermal environment conditions are lower during night and morning, so the effective temperature is less than $20.5^{\circ} \mathrm{C}$ ET. When viewed on the basis of the research site, the outdoor effective temperature is lower than around the river: the open space around the floating market, housing aside pedestrian ways in Bilu, and the floating houses. In two research sites: Sasirangan and Bilu village, the riverfronts are always cooler than in settlement areas, while the open space in Siring Tendean without vegetation is colder than the open space with vegetation. The best outdoor condition and always whithin the comfort zone was the green open space in Siring Tendean (Table 9). Based on the conditions described, the environmental conditions will be better with the vegetation and vegetation planting provides a better effect on the thermal comfort of the environment. The vegetation around the river was also able to withstand the effective temperature not less than $20.5^{\circ} \mathrm{C}$ ET.

The indoor thermal comfort in all research locations during the day has always uncomfortable conditions. The thermal discomfort during the day due to the high effective temperature, exceeds $27.1^{\circ} \mathrm{C}$ ET from around $10 \mathrm{AM}$ to $8 \mathrm{PM}$. The indoor and outdoor thermal comfort occurs at different time (Table 9). Outdoor comfortable conditions occur during the day while indoor at night. The outdoor uncomfortable conditions occur at night because the effective temperature exceeds the lower comfort level limits $\left(17-20.4^{\circ} \mathrm{C}\right.$ ET), while indoor occurs during the day because the effective temperature exceeds the upper comfort level limit $\left(27.2-30^{\circ} \mathrm{C}\right.$ ET). The outdoor thermal comfort conditions around the river are quite good. This is because the river becomes an open space that can drain the air (wind) well. This potential can be further optimized with the rehabilitation and structuring of slum areas. 
TABLE 9. Difference of Thermal Comfort between Indoors and Outdoors on Time

\begin{tabular}{|c|c|c|c|c|}
\hline \multirow{2}{*}{\multicolumn{2}{|c|}{\begin{tabular}{|l|} 
Rank \\
Outdoor
\end{tabular}}} & \multicolumn{3}{|c|}{$\begin{array}{ll}\text { Time } \\
1 \mid 12\left[\left.13\right|_{14}\right.\end{array}$} \\
\hline & & & & \\
\hline \multicolumn{2}{|c|}{\begin{tabular}{l|l}
1 & Green open space
\end{tabular}} & \multirow{7}{*}{$17-20.4^{\circ} \mathrm{C}$ ET } & \multirow{7}{*}{ 20.5-27. ${ }^{\circ} \mathrm{C} \mathrm{ETI}$} & \\
\hline 2 & Menara \& Anno building & & & \\
\hline 3 & Housing in settlement of Sasirangan & & & \\
\hline 4 & Floating houses & & & \\
\hline 5 & Housing in settlement of Bilu & & & \\
\hline 6 & Housing aside pedestrian ways in Bilu & & & \\
\hline 7 & Open space around floating market & & & \\
\hline \multicolumn{2}{|c|}{ Indoor } & & & \\
\hline 1 & Housing in settlement of Bilu & \multirow{5}{*}{ 22.8-27.1 ${ }^{\circ} \mathrm{CET}_{\mathrm{T}}$} & \multirow{5}{*}{ 27.1-30 ${ }^{\circ} \mathrm{C} \mathrm{ET}$} & \multirow{5}{*}{ 22.8-27.10 $\mathrm{CET}$} \\
\hline 2 & Menara \& Anno building & & & \\
\hline 3 & Floating houses & & & \\
\hline 4 & Housing aside pedestrian ways in Bilu & & & \\
\hline 5 & Housing in settlement of Sasirangan & & & \\
\hline - & \begin{tabular}{|l|l|} 
Com fort \\
|Uncouffort
\end{tabular} & & & \\
\hline
\end{tabular}

The outdoor of Menara and Anno are the most comfortable places concerning thermal environment compared to other places in outdoor research buildings. The outdoor comfort conditions of Menara and Anno are influenced by the green open spaces that exist around the building. However, the comfortable outdoor conditions, cannot be used optimally for the indoor thermal comfort. The indoor thermal comforts are not good during the day. The thermal comfort conditions in Menara is better than Anno, caused by the building shape in Menara is rectangular that can maximize indoor air movement. Anno's building is large form, plus the design does not use the cross ventilation system, causing this building to be more uncomfortable. Although the construction of Anno's building is made of wood which is faster in receiving and removing the heat than the concrete material, because the building design does not utilize the cross ventilation system, the heat inside the building does not come out. As a result, the air conditions and fans are used during the day in the building.

The houses indoor in the settlement Bilu village are the most comfortable. This condition is caused by the environment around the house is an open space in the Muslim cemetery. Muslim cemetery is overgrown by vegetation. In addition, the distance between buildings that are not so close to making indoor and outdoor ventilation can circulate well. The influence of the condition of the building is caused by the shape and material of the building. Simple building form with the pattern of the room is also simple, causing air circulation in the house to run smoothly. The presence of ceiling in the house is also able to reduce heat coming from the roof of the building. Building materials made of wooden construction which more quickly absorb and release environmental thermal conditions also affect the thermal comfort in buildings.

Thermal comfort conditions in floating houses and houses aside pedestrian ways have the same conditions. Thermal comfort during the day is uncomfortable because the effective temperatures are greater than $27.1^{\circ} \mathrm{C}$ ET. This condition occurs in 9 hours. The same thermal conditions in these buildings are also due to the same thermal conditions that are in the vicinity of the river. In addition, the shape and character of the building are also the same. The houses on the riverbank have small dimensions. And these houses also do not have a ceiling (Table 10), so the heat from the roof during the day directly affect the thermal environment. The condition is exacerbated by the absence of a good cross-ventilation system in buildings. The roof material made of zinc also adds to the heat of the room, because the zinc material is very hot when exposed to direct sunlight.

TABLE 10. Relationship of Thermal Comfort between The Building Condition and Environment

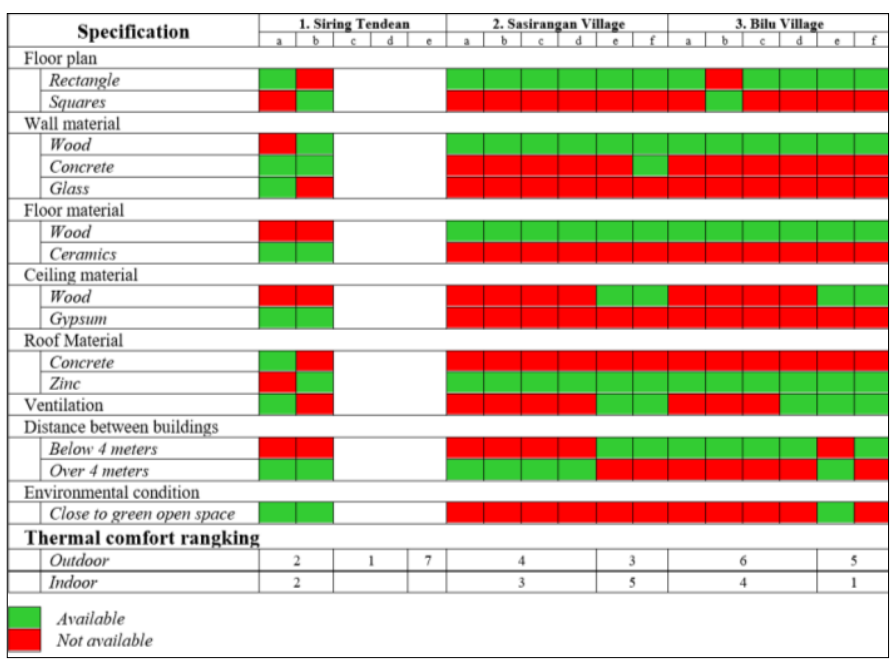

The houses indoor condition in the Sasirangan village settlement is most uncomfortable. This is due to the very close inter-house distance below 4 meters (high house density), causing the ventilation is not good. Air circulation can only be from the front side of building. The large house form with many room bulkheads also causes less ventilation in the building. Renovate the back of house for opening access to the river, so the air can enter into buildings that blow from the river. To support this idea, it is necessary to access pedestrian ways on the riverbank such as in Bilu village. The pedestrian way is designed with due regard to thermal comfort. The pedestrian way design can pay attention to the selection of construction materials, vegetation planting and shading. Shade is significant to protect from heat and rain. The shade materials from wood and vegetation are better and the zinc material as shade should be avoided. This arrangement will also improve the visual quality of the riverside.

\section{CONCLUSIONS AND RECOMENDATIONS}

Thermal comfort of SNI 03-6572 in open space between green open space and the open space without vegetation has the effective temperature difference, either minimal or maximum. Vegetation planted around the building has an effect on the buildings thermal environment. Vegetation around rivers and buildings can lower the effective temperature by $0.7^{\circ} \mathrm{C}$ ET. The difference of minimum effective temperature between green open space and open space without vegetation are $1^{\circ} \mathrm{C}$ ET. The open space without vegetation is lower $1^{\mathrm{O}} \mathrm{C}$ ET than vegetated. Open space without vegetation in uncomfortable condition while vegetated still in comfortable zone SNI 03-6572. Effective temperature in green open space is always in the comfort zone SNI 03 6572 at night. On the other hand, the open space without vegetation around the river is very cold. Effective temperature in green open space is always in the comfort zone during the 
day. On the other hand, the open space without vegetation around the river is hot.

The river is an open space. The open space of the river affects the velocity of the airflow, resulting in higher wind speeds around the river. The value of effective temperature is lower if wind speed is higher. Thermal comfort around the river is influenced by wind movement. Minimum and maximum outdoor relative humidity between riverside buildings and settlements have differences. Minimum relative humidity on the riverside is $4.5 \%$ higher than outdoor settlement. Relative humidity by the river is higher in the morning. The maximum outdoor relative humidity at the riverside is $12 \%$ lower than the outdoor settlement. The relative humidity in settlement is higher on the day.

The condition of indoor buildings in thermal comfort around the river will be explained below:

1. The minimum indoor effective temperature condition is comfortable. On the other hand at the maximum value of effective temperature is hot condition or uncomfortable.

2. The indoor effective temperature has differences of $1^{\mathrm{O}} \mathrm{C}$ ET between riverside buildings and settlement.

3. Minimum and maximum indoor relative humidity between riverside buildings and settlements has differences.

4. Minimum indoor relative humidity at the riverside is $6.5 \%$ higher than outdoor settlement. Relative humidity by the river is higher in the morning.

5. The maximum indoor relative humidity at the riverside is $12 \%$ lower than the outdoor settlement. Relative humidity in higher settlement occurs during the day.

6. The indoor of thermal comfort around the river is the optimal comfort zone SNI 03-6572 from night until morning.

7. The indoor of thermal comfort around the river is uncomfortable zone SNI 03-6572 at during the day.

8. Thermal condition of buildings with ventilation is better than unventilated ones.

9. Buildings on the riverside are largely unventilated so that during the day the effective temperature is high and uncomfortable. While the building of the settlement has ventilation.

10. Effective temperature of buildings with zinc roofing is high during the day. All houses the riverside roofed with zinc, so that during the day is quite hot and uncomfortable.

11. Wood-based buildings are higher in effective temperature than concrete buildings, and the effective temperature difference is about $2^{\circ} \mathrm{C}$ ET.

12. Effective temperature of wooden building is faster than minimum to maximum about 6 hours. At the same time, the building is made of concrete slower about 10 hours.
13. Buildings that have a ceiling is better, because the value of effective temperature is lower than buildings that have no ceiling. All buildings on the riverside have no ceiling so that it is quite hot and uncomfortable.

14. The shape of the rectangular structure is more comfortable with the thermal comfort than the square shape.

15. The distance between buildings affects thermal comfort. A good distance between buildings is over 4 meters.

Research Recommendations will be explained below:

1. Urban development should pay attention to vegetation arrangement. Vegetation has proven to be able to degrade urban heat. Indonesian Regulation Number 05/PRT/M/2008 concerning Guidelines for Provision of Green Open Space in Urban Areas, which already exist should be applied properly [23,24]. A minimum of $30 \%$ of green open space in urban areas can be applied thoroughly in every city area in a balanced manner, not only in certain areas, especially in urban centers that heat the impact of the urban heat island.

2. The land condition is limited in urban areas, then greening can maximize the spaces in the yard of the house. Socialization and community empowerment should be continuously improved to provide awareness of the importance of a comfortable and good environment.

3. The building design must always pay attention to environmental thermal conditions. Building design should be able to take advantage of indoor and outdoor air circulation system well. So that indoor and outdoor air exchange can be maximal.

4. Rehabilitation of houses back to the river by opening access to the river, so the air can enter into buildings that blow from the river. Properly modified buildings will improve the quality of the building [25]. To support the idea, it is necessary to access pedestrian ways on the riverbank such as in Bilu village. Pedestrian paths are designed with thermal comfort. Selection of construction materials, vegetation planting, and shade. Shade is significant to protect from heat and rain.

5. The shade materials from wood and vegetation are better and the zinc material as shade should be avoided

6. Building design should pay attention to air circulation. The goal is to maximize air exchange indoors and impact the thermal comfort.

\section{REFERENCES}

[1] Rapoport, A. (1969). House Form and Culture, Prentice-Hall, Englewood Cliffs, New Jersey.

[2] Lechner, N. (2000). Heating. Cooling, Lighting (Metode Desain Arsitektur), Raja Grafindo Persada, Jakarta

[3] Sari, R., Suzana. (2006). Arsitektur Tropis Bangunan Tradisional Indonesia, Badan Penerbit Universitas Diponegoro, pp. 1-91.

[4] Roth, M. (2013). Handbook of Environmental Fluid Dynamics: Urban Heat Islands. CRS Press/Taylor and Francis Group. Vol. 2: 143-160

[5] Al-Bishawi, M., Ghadban, S. (2011). A Methodological Approach for Reading Urban Open Space. International Journal of Architectural Research: ArchnetIJAR. 5 (1). 73-85.

[6] Karyono, T. (2001). 'Wujud Kota Tropis di Indonesia: suatu pendekatan iklim, lingkungan dan energi', Dimensi Teknik Arsitektur, Vol. 29, No. 2, Desember 2001: 141-146 
[7] Rahman, Akbar. (2008). 'Pengaruh pola pembayangan terhadap kenyamanan jalur pedestrian', Peran Arsitektur Perkotaan dalam Mewujudkan Kota Tropis, UNDIP. Semarang.

[8] Rahman, Akbar. (2010). 'The Different Air Temperature in The Shadowed and Unshadowed Condition, International Conference on Sustainable Environmental Architecture (SENVAR), ITS, Surabaya.

[9] Iswanto, D. (2006). Pengaruh Elemen-elemen Pelengkap Jalur Pedestrian terhadap Kenyamanan Pejalan Kaki, Enclosure.

[10] Tisnaningtyas, E. (2015). 'Aspek Kenyamanan Pejalan Kaki terhadap Iklim Tropis dan Aksesibilitas Pejalan Kaki di Lapangan Kota sebagai Kawasan Pedestrian', Journal of Architecture, Pandanaran.

[11] Lippsmeier, G. (1997) Bangunan Tropis, Erlangga, Jakarta.

[12] Bromberek Z. (2007). An Argument Againts Air Conditioning Use in Tropical Resorts. International Journal of Architectural Research: ArchnetIJAR. 1 (3). 115-126.

[13] Sahabuddin M., and Gonzalez-Longo C. (2015). Traditional Values and Their Adaptation in Social Housing Design: Towards a New Typology and Establishment of 'Air House' Standard in Malaysia. International Journal of Architectural Research: ArchnetIJAR. 9 (2). 31-44.

[14] Rahman, Akbar, Kojima, Shoichi. (2017). Analysis of thermal comfort SNI-6390 in the Lanting (floating house). International Proceedings of Chemical, Biological and Environmental Engineering, Vol. 100.

[15] Rahman, Akbar, Kojima, Shoichi. (2017). Study of Indoor and Outdoor Thermal Comfort for Public Space and Houses in Around River, Case Study: Banjarmasin City, Indonesia. Advances in Engineering: an International Journal (ADEIJ). Vol. 2 (1). 29-41. DOI:10.5281/zenodo.1244077.

[16] Rahman, Akbar, Kojima, Shoichi. (2017). Analysis of Thermal Comfort SNI 03-6572 In Green Open Space Siring Tendean Banjarmasin-Indonesia. 6th International Conference on Innovation in Civil, Architecture, Environment and Materials Engineering (CAEME17) Paris (France). Oct. 5-6, 2017. pp. 40-44. DOI: 10.17758/EIRAI

[17] ASHRAE Standard 55 P: Thermal Environmental Conditions for Human Occupancy, 2003.

[18] Badan Standardisasi Nasional (BSN). (2001). Standar Nasional Indonesia (Indonesian National Standardization)-SNI 03-6572:2001 Tata Cara Perancangan Sistem Ventilasi dan Pengkondisian Udara pada Bangunan Gedung, BSN, Jakarta, Indonesia.

[19] Badan Standardisasi Nasional (BSN). (2011). Standar Nasional Indonesia (Indonesian National Standardization)-SNI 6390:2011 Konservasi Energi Sistem Tata Udara Bangunan Gedung, BSN, Jakarta, Indonesia.

[20] Tharziansyah, Muhammad, Rahman, Akbar (2008). Analisis Tingkat Kenyamanan Thermal Webb Di Rumah Tinggal T-45 Pada Musim Kemarau. Infoteknik, Vol. 9, 36-42.

[21] Zakariya K., Kamarudin Z., and Harun Z. N. (2016). Sustaining the Cultural Vitality of Urban Public Market: A case study of Pasar Payang, Malaysia. International Journal of Architectural Research: ArchnetIJAR. 10 (1). 228-239.

[22] Agboola O., Rasidi M., Said I. (2017). The Influence of Open Space Utilization on Residents Attachment with Community: A Case Study of Rural Market Square in Southwest Nigeria. International Journal of Architectural Research: ArchnetIJAR. 11 (1). 44-66.

[23] PU. 'Peraturan Menteri PU No. 05/PRT/M/2008 tentang Pedoman Penyediaan Ruang Terbuka Hijau di Kawasan Perkotaan'. accessed on June $7^{\text {th }} ， \quad 2017$, <http://www.penataanruang.com/pedoman-ruangterbuka-hijau.html>.

[24] BNPB. 'Undang-undang tentang Penataan Ruang'. accessed on Juni $7^{\text {th }}$, 2017, <https://www.bnpb.go.id/uploads/migration/pubs/2.pdf >.

[25] Carnemolla P., Bridge C. (2016). Accessible Housing and HealthRelated Quality of Life: Measurements of Wellbeing Outcomes Following Home Modifications. International Journal of Architectural Research: ArchnetIJAR. 10 (2). 38-51. 